\title{
Lessons Learnt after the Introduction of the Seven Valent-Pneumococcal Conjugate Vaccine Toward Broader Spectrum Conjugate Vaccines
}

\author{
Oana Falup-Pecurariu
}

\begin{abstract}
The 7-valent pneumococcal conjugate vaccine (PCV7) is currently being introduced in the vaccine schedule of over 90 countries around the world. After the introduction of the PCV7 vaccine in the United States, a reduction of more than $90 \%$ of invasive pneumococcal disease (IPD) was reported in vaccinated children under the age of 5 years. Similar findings were reported from other countries. A reduction in community-acquired pneumonia (CAP) of $>40 \%$ has also been reported. In children under the age of 5 years, the number of primary medical visits and antibiotic usage for acute otitis media (AOM) decreased by more than $40 \%$. In adults over 65 years of age a significant reduction of $90 \%$ in IPD caused by PCV7 serotypes was reported as well. However, after the introduction of PCV7 there were reports of increase of serotypes not included in the vaccine, such as serotype 19A in various Streptococcus pneumoniae-related diseases such as invasive disease, AOM and

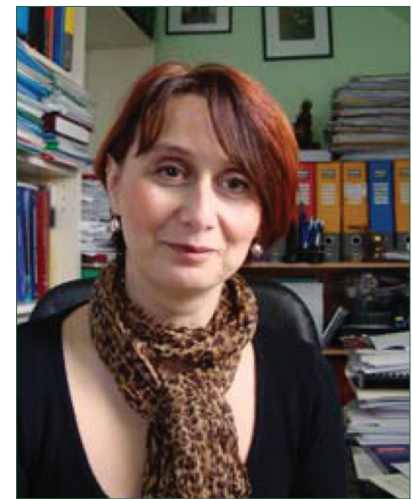

Dr. Oana Falup-Pecurariu pneumonia. In addition, serotypes 1, 5, 7F and 19A were more prevalent in complicated cases of CAP. Recently, new vaccines covering additional serotypes such as the 10-valent pneumococcal conjugate vaccine (PCV10) and 13-valent pneumococcal conjugate vaccine (PCV13) were introduced, and are expected to reduce $S$. pneumoniae-related diseases furthermore. (Biomed J 2012;35:450-56)
\end{abstract}

Key words: acute otitis media, children, community-acquired pneumonia, invasive pneumococcal disease, pneumococcal conjugated vaccine (PCV7), vaccine serotypes

Ctreptococcus pneumoniae remains an important cause Oof serious illness such as pneumonia, meningitis, bacteremia among children and adults worldwide. It is also a major cause of sinusitis and acute otitis media (AOM). ${ }^{[1]}$

In double-blind randomized case-control clinical trials, the 7-valent pneumococcal conjugate vaccine (PCV7) was demonstrated to be efficacious and safe against invasive pneumococcal disease (IPD). In adition, PCV7 was demonstrated to prevent $20-37 \%$ of community-acquired alveolar pneumonia in children and approximately $56 \%$ of acute otitis media caused by serotypes in included in PCV7. ${ }^{[1]}$

In 2000, a 7-valent pneumococcal polysaccharide-protein conjugate vaccine (PCV7; Prevnar, Wyeth) was licensed by the Food and Drug Administration (FDA) for use among infants and young children in the United States. ${ }^{[2]}$ Since its introduc- tion in North America, PCV7 has been licensed in over 90 countries and has contributed to a massive decline in diseases caused by $S$. pneumoniae. ${ }^{[1]}$ However, after PCV7 introduction several reports emerged on the increased incidence of other serotypes not included in the PCV7 such as serotype 19A in IPD and in AOM. ${ }^{[3]}$ Moreover, the role of additional non-PCV7 vaccine serotypes, mainly serotypes $1,3,5$, and $7 \mathrm{~F}$ was shown both in invasive diseases and in mucosal diseases such as community-acquired pneumonia (CAP) and AOM. ${ }^{[4-8]}$

Recently two new broader spectrum vaccines were introduced: 1) A 10-valent vaccine conjugated to a D protein obtained from Haemophilus (the innovative PHiD-CV) contains the 7 serotypes included in PCV7 and in addition serotypes 1,5 and $7 \mathrm{~F} ; 2$ ) the 13 -valent pneumococcal polysaccharideprotein conjugate vaccine (PCV13) containing the

From the Department of Pediatrics, University Childrens' Hospital, Transilvania University, Brasov, Romania.

Received: Feb. 4, 2012; Accepted: May 7, 2012

Correspondence to: Dr. Oana Falup-Pecurariu, Department of Pediatrics, University Childrens' Hospital, Transilvania University. B-dul Eroilor No. 29, Zip Code 500036, Brasov, Romania. Tel: 40-268-413000; Fax: 40-268-418988; E-mail: oanafp@yahoo.co.uk

DOI: $10.4103 / 2319-4170.104409$ 
7 serotypes included in PCV7 and 6 additional pneumococcal conjugate serotypes including 1, 3, 5, 6A, 7F, and 19A. These vaccines are expected to increase the covarage of pneumococcal disease and to reduce significantly more the burden of IPD, AOM and CAP world wide.

\section{The effect of PCV7 on invasive disease}

The introduction of PCV7 into routine infant immunization programs has led to the near eradication of vaccine-serotype IPD in vaccinated children as well as in older children and adults, through herd protection. ${ }^{[9-11]}$ Data from different studies show that the incidence of IPD due to PCV7 vaccine serotypes in children $<5$ years of age has declined by $>90 \%$. The rate of invasive pneumococcal disease for children under 2-years old declined by $76 \%$ by 2007 , compared with prevaccine rates; the decline for infections caused by serotypes included in the vaccine was $100 \% .{ }^{[12]}$ Although Afro-American children remain at higher risk of IPD, the introduction of childhood pneumococcal vaccination has reduced the racial disparity in incidence of pneumococcal disease. ${ }^{[13]}$

Moreover, after the introduction of PCV7, there has been a significant decline in IPD in adults, in particular in older adults ( $>65$ years old). Data from the United States show a $73 \%$ reduction in IPD due to the PCV7 serotypes in adults 65 years of age and older. The absolute number of cases prevented by this indirect effect of PCV7 is actually estimated to be larger than the direct effect, which makes universal vaccination highly cost effective. ${ }^{[12]}$ The reductions were due to decreases in vaccine-type disease (92\%) and are thought to be related to reduced transmission of vaccine serotype among children, a phenomenon named herd protection (immunity), which can also be called indirect effect. ${ }^{[14]}$ Thus, in many areas of the United States, substantial reductions in invasive pneumococcal infections occurred despite the shortage of PCV7 beginning in the fall of 2001-2002, which may have modified the reduction in cases. These reduction rates were also observed in other countries such as Europe, Canada and Australia after the introduction of PCV7 to their vaccine schedules. ${ }^{[15]}$

\section{Serotypes replacement after the introduction of PCV7}

Recent reports have pointed out an increase in the non-vaccine serotypes. ${ }^{[16]}$ In 2008 , a total of $61 \%$ of IPD cases among children aged $<5$ years were attributable to the serotypes covered in PCV13, with serotype 19A accounting for $43 \%$ of cases; PCV7 serotypes caused $<2 \%$ of cases. Three of the six additional serotypes, (19A, 7F, and 3) accounted for $99 \%$ of IPD cases, serotypes 1 and 5 together caused $0.6 \%$ of cases, and serotype $6 \mathrm{~A}$ caused $0.6 \%$ of cases. In the age group $\geq 5$ years, the serotypes included in
PCV7 and PCV13 caused 4-7\%, and 43-66\% of IPD cases, respectively. Following routine PCV7 use, the incidence of IPD caused by penicillin-resistant strains decreased by $57 \%$ overall and by $81 \%$ among children aged $<2$ years. ${ }^{[16]}$

Invasive pneumococcal disease caused by penicillin-nonsusceptible non-PCV7 serotypes increased, and most of the resistant infections are now caused by serotype 19A. ${ }^{[17]}$ Between 2007 and 2009 following PCV7 implementation in Spain, a surveillance study was undertaken in order to assess all isolates from invasive disease. ${ }^{[18]}$ The most common serotypes isolated from IPD cases were serotypes included in the PCV13 (but not in PCV7): 19A (26.1\%), 5 (18.8\%), $7 \mathrm{~F}(8.5 \%)$ and $3(3.9 \%)$ pointing out the benefits of introducing this vaccine in Spain. ${ }^{[19]}$ In a 2-year Spanish survey on invasive disease, 19A was the most common encountered serotype. ${ }^{[18]}$ In the Navajo population, between 1995 and 2006, in an active surveillance system which included 1508 cases of IPD the total number of cases decreased from 67 in 1995-1997 to 51 cases in the years following PCV7 introduction. ${ }^{[20]}$ There was no change in the nonvaccine type (NVT) in children in the post vaccine era. Rates increased for serotypes 1, 3, 5, 7F, 19A, 18B. In infants under the age of 2 months no cases with serotype included in the PCV7 was reported with no replacement by NVT disease. ${ }^{[20]} \mathrm{A}$ combined study of four European countries was done after PCV7 introduction in Europe. ${ }^{[21]}$ Different countries have implemented vaccination in various time periods, but all consider the 7 serotypes contained in the vaccine as being responsible for $68 \%$ to $77 \%$ of IPD in children under 5 years of age. During 2005 and 2006 the incidence of IPD started to increase due to NVT both in the under 5-year-old population and children 5-14 years old. ${ }^{[22]}$ The most encountered serotypes were: $1,7 \mathrm{~F}$ and $19 \mathrm{~A}$ considered to be nonvaccine serotypes. However, for children under the age of 5 years, serotype 1 started to become more prevalent before PCV7 introduction. Moreover, two peak point increases betwen 1996-2002 and 2005- 2006 were reported. For the 5-14 years old group the major cause of IPD in 50\% of cases was serotype 1 in 2005-2006. For serotype 7F most cases occured in the age group under 5 years and increased mostly during 2004-2006 except in Spain. For 2005-2006 it increased mostly in Spain but less so in England and Wales. With regard to serotype 19A incidence of IPD actually doubled in the group of the under 5 years of age. ${ }^{[21]}$

While for countries having adopted PCV7, the prevalence of serotypes 1,7F and 19A increased, only serotype 1 increased in England and Wales. Antimicrobial resistance pattern showed high resistance rates for Spain, Belgium and France after the introduction of PCV7. The resistance rates increased to $86 \%$ for penicillin and around $77 \%$ for macrolides. There may be other explanations besides that of PCV7 selective pressure such as the overuse of macrolides 
with selective pressure toward more antibiotic resistant isolates especially for serotype 19A. Another explanation is the secular trend or the cyclical trend of serotype 1 .

A study from Portugal on invasive disease during 2006-2008 reported that serotypes included in the PCV7 accounted for only $17 \%$ of the cases. The most frequent causes of IPD were due to serotypes $1,7 \mathrm{~F}$ and 19A. ${ }^{[23,24]}$

In an observational study from Dallas, TX, USA, conducted over a period of 8 years (1999-2008) and comparing the two periods before and after PCV7 introduction, a decrease in IPD during 2002-2008 was reported. However, between 2006 and 2008 the incidence of IPD started to increase. Serotype 19A was the one that increased significantly and its resistance to antibiotic drugs such as penicillin and cefotaxime significantly increased as well. The increase in the resistance rate of serotype 19A was due to the introduction of clonal complex cc-156 and cc-320 which emerged at the same time as 19A resistance to penicillin and cefotaxim. ${ }^{[25]}$

\section{The effect of PCV7 on CAP}

Six trials tested the effect of vaccine on clinically and radiologically diagnosed CAP. In the Northern California trial, vaccination reduced episodes of pneumonia confirmed by radiography by $30 \% .{ }^{[26]}$ A study among children without HIV infection in South Africa showed that the vaccine reduced the first episodes of alveolar pneumonia by $25 \%$ among fully immunized infants. ${ }^{[27]}$ In the Gambia, vaccination reduced radiologically confirmed pneumonia by $37 \%$, including a reduction of hospital admissions and all-cause mortality by $15 \%$ and $16 \%$, respectively. ${ }^{[28]}$ The American Indian trial pneumonia results were inconclusive the performance of the vaccine may vary in accordance to many aspects including epidemiological and population characteristics. ${ }^{[29]}$ Subsequently an investigational 11-valent conjugate vaccine reduced alveolar consolidation confirmed pneumonia by $23 \%$ in the Philippines ${ }^{[30]}$ and a 10 -valent conjugate including serotypes conjugated to Haemophilus protein $\mathrm{D}$ has been reported to reduce pneumonia in children in South and Central America by $25 \% .^{[31]}$

Since the introduction of PCV7, a reduction in CAP of $39 \%$ and primary physician visits due to AOM of $42 \%$ were reported. ${ }^{[32,33]}$ Beside the serotypes included in PCV7, the most common serotypes causing IPD are 1 and 5, prevalent mainly in developing countries, ${ }^{[34,35]}$ and particularly in $\mathrm{Asia}^{[36]}$ where the incidence rates of these diseases are significantly higher compared with North America and Europe. ${ }^{[37,38]}$ These serotypes have also been reported in outbreaks of IPD and CAP in both children and adults. ${ }^{[39-41]}$ Serotype 1 is common in children $>2$ years of age and in complicated pneumonia cases. ${ }^{[42]}$ Following the introduction of the PCV7, significant impact on CAP was demonstrated showing up to $39 \%$ reduction in all-cause pneumonia. ${ }^{[43]}$ However, increasing rates of empyema have been reported from various countries mainly caused by serotypes 1, 3, 5, 7F and 19A (serotypes not included in the vaccine) ${ }^{[4]}$ The disease potential of such serotypes as 1,5 and $7 \mathrm{~F}$ have been reported significantly more often in IPD, CAP and AOM. ${ }^{[44]}$

In Poland, the two infant doses schedule of PCV7 was found to be efficacious for reduction of CAP in children. ${ }^{[45]}$

A significant impact of pediatric vaccination on reduction in hospitalization for alveolar pneumonia in adults in the US has been recently shown and more than $90 \%$ of the reduction in hospitalization for pneumonia attributable to the vaccine is the reduction in disease in adults due to herd protection. ${ }^{[46]}$

\section{The effect of PCV7 on AOM}

In a randomized clinical trial conducted in Finland in which the bacterial etiology of AOM was determined by myringotomy, the efficacy of PCV7 in preventing culture-confirmed, vaccine serotype AOM episodes was 57\% (95\% CI, 44-67\%): the overall net reduction in AOM caused by any pneumococcal serotype was $34 \%$ (95\% CI, 21-45\%). Overall, PCV7 prevented 6-7\% of all AOM episodes in the clinical trials, ${ }^{[47]}$ reductions also were observed for the outcomes of frequent otitis media (9\%) and tympanostomy tube placement $(20 \%){ }^{[48]}$

Following the introduction of PCV7 in the United States, a reduction of $20 \%$ in otitis media visit rates in children under 2 years of age was reported. This decline represented 246 fewer otitis media visits per 1000 children in this age group annually. ${ }^{[49]}$ These finding were similar in a population base study in Quebec reporting a reduction of physician claims for otitis media attributable to PCV-7 of $13.2 \%{ }^{[50]}$ In Australia, 2.5 years after the introduction of PCV7 a significant adjusted decrease in myringotomy with ventilation tube insertion in children aged under 1, 1-2, and older than 2-years old of $23 \%, 16 \%$, and $6 \%$, respectively, ${ }^{[51]}$ was reported. Similar findings were reported in Tennessee in children under 2 years of age with reductions of $17 \%$ in AOM rates and $23 \%$ in pressure-equalizing tube insertions. In New York decline rates of $16 \%$ and $23 \%$ were reported in $\mathrm{AOM}$ and pressure-equalizing tube insertions when the 2000-2001 birth cohort (after PCV7 introduced) was compared to the 1998-1999 birth cohort (pre-PCV7 vaccination period). ${ }^{[52]}$ Reduction in hospitalization rates in children under 2 years of age with AOM was reported from Liguria, Italy. ${ }^{[53]}$

After the introduction of PCV7 into the national immunization program in United States, rates of ambulatory visits and antibiotic prescriptions attributable to AOM decreased by $42.7 \%$ and $41.9 \%$, respectively. ${ }^{[54]}$ Total estimated nation- 
al direct medical expenditures for AOM-related ambulatory visits and antibiotic prescriptions for children younger than 2 years of age decreased from an average of $\$ 1.41$ billion during 1997 to 1999 to $\$ 0.95$ billion in 2004 (32.3\% reduction). ${ }^{[54]}$ This reduction was sustained and a $36 \%$ reduction in antibiotic prescription was also reported after 6 years of PCV7 usage in the United states. ${ }^{[5]}$ In a study of Hoberman et al. ${ }^{[56]}$ it was shown that the proportion of the $S$. pneumoniae that were vaccine serotypes declined progressively while there was an increase of penicillin non susceptible isolates with 19A being one of the most important. Also it was shown that the penicillin nonsusceptible isolates belonged to $19 \mathrm{~A} .{ }^{[56]}$

\section{The effect of PCV7 on nasopharyngeal carriage}

Pneumococcal conjugate vaccines have been shown to reduce the risk of carriage and transmission of vaccine-type strains among vaccines and their household members. PCV7 reduces the acquisition of vaccine-type strains but increases the risk for carriage of nonvaccine serotype. Several studies have found an increase in nasopharyngeal colonization by nonvaccine or replacement serotypes of $S$. pneumoniae among children who received a conjugate pneumococcal vaccine. ${ }^{[57,58]} \mathrm{In}$ addition to the PCV7, the 9-valent vaccine conjugated to CRM197 reduced nasopharyngeal pneumococcal carriage in toddlers, especially in those less than 36 months of age. ${ }^{[59]}$

In a study in the pre-PCV7 period, looking at the carriage of $S$. pneumoniae serotypes in children $<5$ years old with CAP versus healthy controls it was found that serotypes 1, 5, 7F, 9V, 14, 19A and 22F have a significant relative higher disease potential than serotypes $6 \mathrm{~A}, 6 \mathrm{~B}, 23 \mathrm{~A}$ and $35 \mathrm{~B}$ to cause $\mathrm{CAP} \cdot{ }^{[44]}$ The authors pointed out that the carriage rate was the highest in the 6-17-month-old group. The article suggested that expanding the number of the serotypes in a 13-valent vaccine would provide significantly higher coverage of the more virulent serotypes. ${ }^{[4]}$

In the northern part of Europe, studies looking at carriage were conducted before and after the implementation of a $2+1$ schedule of PCV7 vaccination. In this study 1,213 children were included before and 602 after implementation of PCV7. Despite a decrease in the serotype covered by the vaccine the overall carriage rate remained high. Serotypes included in the PCV7 were carried by $12.7 \%$ of the 237 children who had received at least two doses of vaccine and by $23.3 \%$ of the 365 who received at least one dose of vaccine. Carriage decreased significantly in the group that received at least two doses of vaccine. There was also a decrease of the serotypes included in the vaccine including serotype $6 \mathrm{~A}$, except for serotypes 4 and 19F. However the prevalence of 19A remained the same. ${ }^{[60]}$ In a study by Hoberman et al., where nasopharyngeal samples were obtained from chil- dren aged 6-23 month with AOM divided into three groups pre-PCV7, immediately post- PCV7 and late post-PCV7, the prevalence of the $\mathrm{PCV} 7$ serotypes declined progressively while the non-PCV7 serotypes which were not penicillin nonsusceptible increased, mainly for serotype 19A, 4\% and $25.9 \%$, respectively. ${ }^{[56]}$

\section{Emergence of the new serotypes}

Serotype 6C is considered to be one of the replacement serotypes in the post PCV7 era despite the fact that it may have originated 27 years ago. Recently a new serotype, $6 \mathrm{D}$ was reported. Serotype $6 \mathrm{C}$ originally mutated from $6 \mathrm{~A}$ and serotype $6 \mathrm{D}$ originally mutated from serotype $6 \mathrm{~B} \cdot{ }^{[61]} \mathrm{A}$ specific anti-serum was developed to differentiate among the 6 serogroups (A, B, C, and D). This specific antibodies test was validated using 189 isolates of serogroup 6 obtained from a surveillance study from the United States and Israel. ${ }^{[61]}$

Millar et al., performed a study in the Navajo population in order to assess the epidemiology of serotype $6 \mathrm{C}$ before and after PCV7 introduction. They analysed the 6A serotypes using conventional methods to study $S$. pneumoniae isolates obtained from invasive disease and also triplex polymerase chain reaction to solve the problem of serotypes 6A and 6C. Serotype 6C had a mean annual incidence of $0.3 \%$ and $76 \%$ of the $6 \mathrm{~A}$ isolates causing IPD were $6 \mathrm{C}$ and nearly all were found in adults. ${ }^{[62]}$

Several studies reported that serotype $6 \mathrm{C}$ also circulated in the era pre-PCV7 introduction but was serotyped as serotype $6 \mathrm{~A}$ by the Quellung reaction. In a study performed before PCV7 introduction including over 1,396 presumed serotype $6 \mathrm{~A}$ isolates isolated from blood or cerebrospinal fluid, middle year fluid (MEF) nasopharynx (NP) and conjunctiva from children, serotype $6 \mathrm{C}$ constituted $8.2 \%$ of the presumed serotype $6 \mathrm{~A}$ isolates and had the following distribution, $7.4 \%$ from blood, $3.1 \%$ from MEF, $6.0 \%$ from conjunctiva, $9.8 \%$ from NP cultures. ${ }^{[63]}$

The newly $11 \mathrm{E}$ arises from antigenically similar $11 \mathrm{~A}$ progenitors, and each strain contains a distinct wcjE suggesting that it is not transmitted among hosts.It may independently arise during infection after initial colonization with a $11 \mathrm{~A}$ progenitor serotype. More studies are needed before we could make a clear statement. ${ }^{[64]}$

The pneumococcus is a naturally transformable organism and it has the ability to take up the DNA from other strains and incorporate it into its own genome.

Except for 19A the most common ST in 15B/C strains remains 199 but the emergency of $15 \mathrm{~B} / \mathrm{C}$ has been due to multiple unrelated clones and there is also a marked clonal diversity between different years of study as were 20042007. ${ }^{[65]}$ 


\section{Broader spectrum pneumococcal conjugated vaccines}

A 10-valent vaccine conjugated to a D protein obtained from Haemophilus (the innovative PHiD-CV) was developed. This vaccine contains the 7 serotypes included in PCV7 and in addition serotypes 1, 5 and 7F. It has a robust clinical profile to offer broad protection against IPD and efficacy similar to that of PCV7 for CAP. In addition, this vaccine demonstrated significantly better efficacy than PCV7 for AOM in children. ${ }^{[66]}$

An even broader spectrum PCV was introduced recently worldwide, the PCV13 containing the 7 serotypes included in PCV7 and 6 additional pneumococcal conjugate serotypes including 1, 3, 5, 6A, 7F, and 19A. The addition of these 6 serotypes will potentially increase the percentage of vaccine-preventable IPD cases to $90 \%$ or more in most regions of the world. ${ }^{[65]}$

PCV13 is currently replacing PCV7 in North America, Western Europe, Israel and other countries world wide.

\section{Conclusions}

Since the licensure of PCV7 in the United States in 2000, its effectiveness in preventing IPD in infants has been clearly observed. Data from different studies show that the incidence of IPD due to PCV7 vaccine serotypes in children $<5$ years of age has declined by $>90 \%$. These reduction rates were also observed in other countries such as Europe, Canada and Australia after the introduction of PCV7 to their vaccine schedules. In addition, reduction in CAP and otitis media in children $<5$ years of age were reported from different countries using PCV7. The impact of PCV7 on nasopharyngeal carriage and the resulting indirect effect on herd protection contribute significantly to the cost effectiveness of the vaccine. There was a statistically significant decline in IPD in adults aged 20 years and older after the introduction of PCV7, the most significant in older adults ( $>65$ years old).

However, recent data from United States and Europe have shown increases in IPD caused by non-PCV7 serotypes in both infants and adults. This increase in non-PCV7 vaccine serotypes was mainly observed due to serotype 19A which is characterized by its antibiotic resistance profile. Thus, the dual effect of high antibiotic usage in the community and the usage of PCV7 facilitates increase rates of this serotype worldwide. In addition increased rates of complicated pneumonia in children due to serotypes 1, 3, 5, 7F, and 19A in Europe and North America have been reported.

A broader protection of PCV13 against IPD, pneumonia, and otitis media is anticipated compared to PCV7. In addition, it is anticipated that the PCV13 vaccine will reduce nasopharyngeal carriage of the 6 additional serotypes (1, 3, 5, 6A, 7F and, 19A), and thereby extend herd immunity.

\section{REFERENCES}

1. Dagan R, Greenberg D, Jacobs MR, Lane Phillips B. Pneumococcal Infections. Textbook of Pediatric Infectious Diseases. In: Feigin RD, Cherry JD, Demmler-Harrison GJ, Kaplan S, editors. Vol. 1, $6^{\text {th }}$ ed. Philadelphia: Saunders Elsevier; 2009. p. 1288-342.

2. CDC. Preventing pneumococcal disease among infants and young children: Recommendations of the Advisory Committee on Immunization Practices (ACIP). MMWR Morb Mortal Wkly Rep 2000;49; RR-9).

3. Pichichero ME, Casey JR. Emergence of a multiresistant serotype 19A pneumococcal strain not included in the 7-valent conjugate vaccine as an otopathogen in children. JAMA 2007;298:1772-8.

4. Obando I, Muñoz-Almagro C, Arroyo LA, Tarrago D, Sanchez-Tatay D, Moreno-Perez D, et al. Pediatric parapneumonic empyema, Spain. Emerg Infect Dis 2008;14:1390-7.

5. Bekri H, Cohen R, Varon E, Madhi F, Gire R, Guillot F, et al. Streptococcus pneumoniae serotypes involved in children with pleural empyemas in France. Arch Pédiatr 2007;14:239-43.

6. Hortal M, Sehabiague G, Camou T, Iraola I, Estevan M, Pujadas M. Pneumococcal pneumonia in hospitalized Uruguayan children and potential prevention with different vaccine formulations. J Pediatr $2008 ; 152: 850-3$.

7. Copelovitch L and Kaplan SB. Streptococcus pneumoniae-associated hemolytic uremic syndrome: classification and emergence of serotype 19A. Pediatrics 2010;125:e174-82.

8. Waters AM, Kerecuk L, Luk D, Haq MR, Fitzpatrick MM, Gilbert RD, et al. Hemolytic uremic syndrome associated with invasive pneumococcal disease: the United Kingdom experience. J Pediatr 2007;151:140-4.

9. Whitney CG, Farley MM, Hadler J, Harrison LH, Bennett NM, Lynfield R, et al. Decline in invasive pneumococcal disease after the introduction of protein-polysaccharide conjugate vaccine. N Engl J Med 2003;348:1737-46.

10. Kellner JD, Vanderkooi OG, MacDonald J, Church DL, Tyrrell GJ, Scheifele DW. Changing epidemiology of invasive pneumococcal disease in Canada, 1998-2007: update from the Calgary-area Streptococcus pneumoniae research (CASPER) study. Clin Infect Dis 2009;49:205-12.

11. Black SB, Shinefield HR, Hansen J, Elvin L, Laufer D, Malinoski F. Postlicensure evaluation of the effectiveness of seven valent pneumococcal vaccine. Pediatr Infect Dis J 2001;20:1105-7.

12. Pilishvili T, Lexau C, Farley MM, Hadler J, Harrison LH, Bennett NM, et al. Sustained reductions in invasive pneumococcal disease in the era of conjugate vaccine. J Infect Dis 2010;201:32-41.

13. Flannery B, Schrag S, Bennett NM, Lynfield R, Harrison LH, Reingold $\mathrm{A}$, et al. Impact of childhood vaccination on racial disparities in invasive Streptococcus pneumoniae infections. JAMA 2004;291:2197-203.

14. Centers for Disease Control and Prevention (CDC). Direct and indirect effects of routine vaccination of children with 7-valent pneumococcal conjugate vaccine on incidence of invasive pneumococcal disease- United States, 1998-2003. MMWR Morb Mortal Wkly Rep 2005;54:893-7.

15. Lepoutre A, Varon E, Georges S, Gutman L, Levy-Bruhl D. Impact of infant pneumococcal vaccination on invasive pneumococcal disease in France, 2001-2006. Available from: http://www.eurosurveillance. 
org/images/dynamic/EE/V13N35/art18962.pdf) [Last accessed on 2012 Jan 27].

16. Jardine A, Menzies RI, McIntyre P. Reduction in hospitalizations for pneumonia associated with the introduction of a pneumococcal conjugate vaccination schedule without a booster dose in Australia. Pediatr Infect Dis J 2010;7:607-12.

17. Centers for Disease Control and Prevention, Prevention of Pneumococcal disease among infants and children-use of 13-valent pneumococcal conjugate vaccine and 23 -valent pneumococcal polysaccharide vaccine. Recommendations of the Advisory Committee on Imunization Practices (ACIP) MMWR 2010, 59 (No. RR -11): 1-19.

18. Picazo J, Ruiz-Contreras J, Hernandez B, Sanz F, Gutierrez A, Cercenado E, et al. Clonal and clinical profile of Streptococcus pneumoniae serotype 19A causing pediatric invasive infections: a 2-year (2007-2009) laboratory based surveillance in Madrid. Vaccine 2011;29:1770-6.

19. Picazo J, Ruiz-Contreras J, Casado- Flores J, Giangaspro E, Del Castillo F, Hernández-Sampelayo T, et al. Relationship between serotypes, age, and clinical presentation of invasive pneumococcal disease in Madrid, Spain, after introduction of the 7-valent pneumococcal conjugate vaccine into the vaccination calendar. Clin Vaccine Immunol 2011;18: 89-94.

20. Weatherholtz R, Millar EV, Moulton LH, Reid R, Rudolph K, Santosham $\mathrm{M}$, et al. Invasive pneumococcal disease a decade after pneum-ococcal conjugate vaccine use in an American Indian population at risk for disease. Clin Infect Dis 2010;50:1238-46.

21. Hanquet G, Kissling E, Fenoll A, George R, Lepoutre A, Lernout T, et al. Pneumococcal serotypes in children in 4 European countries. Emerg Infect Dis 2010;16:1428-39.

22. Kaplan SL, Barson WJ, Lin PL, Stovall SH, Bradley JS, Tan TQ, et al. Serotype 19A is the most common serotype causing invasive pneumococcal infections in children. Pediatrics 2010;125:429-36.

23. Aguilar SI, Brito MJ, Goncalo-Marques J, Melo-Cristino J, Ramirez M. Serotypes 1, 7F and 19A became the leading cause of pediatric invasive pneumococcal infections in Portugal after 7 years of heptavalent conjugate vaccine use. Vaccine 2010;28:5167-73.

24. Gertz RE Jr, Li Z, Pimenta FC, Jackson D, Juni BA, Lynfield R, et al. Increased penicillin non susceptibility of non vaccine serotype invasive pneumococci other than 19A and 6A in the post-7-valent conjugate vaccine era. J Infect Dis 2010;201:770-5.

25. Techasaensiri C, Messina AF, Katz K, Ahmad N, Huang R, McCracken GH Jr. Epidemiology and evolution of invasive pneumococcal disease caused by multidrug resistant serotype-s of 19A in the 8 years after implementation of pneumococcal conjugate vaccine immuni-zation in Dallas, Texas. Pediatr Infect Dis J 2010;29:294-300.

26. Hansen J, Black S, Shinefield H, Cherian T, Benson J, Fireman B, et al. Effectiveness of heptavalent pneumococcal conjugate vaccine in children younger than 5 years of age for prevention of pneumonia: updated analysis using World Health Organiziation standar-dized interpretation of chest radiographs. Pediatr Inf Dis J 2006;25:779-81.

27. Madhi SA, Albrich W. WHO guidelines for treatment of severe pneumonia. Lancet 2007;370:386-7.

28. Cutts FT, Zaman SM, Enwere G, Jaffar S, Levine OS, Okoko JB, et al. Efficacy of nine valent pneumococcal conjugate vaccine against pneumonia and invasive pneumococcal disease in The Gambia: Randomised, double blind, placebo controlled trial. Lancet 2005;365:1139-46.
29. O'Brien KL, Moulton LH, Reid R, Weatherholtz R, Oski J, Brown L, et al. Efficacy and safety of seven valent conjugate pneumococcal vaccine in American Indian children: Group randomised trial. Lancet 2003;362:355-61.

30. Lucero MG, Nohynek H, Williams G, Tallo V, Simões EA, Lupisan S, et al. Efficacy of an 11-valent pneumococcal conjugate vaccine against radiologically confirmed pneumonia among children less than 2 years of age in the Philippines: A randomized, double blind, placebo controlled trial. Pediatr Infect Dis J 2009;28:455-62.

31. Tregnaghi MW, Saez-Llorens X, Lopez P, Abate H, Smith E, Posleman A, et al. Evaluating the efficacy of 10-valent Pneumococcal non-typeable Haemophilus influenzae protein-D conjugate vaccine (PHiD-CV) against community-acquired pneumonia in Latin America. The $29^{\text {th }}$ Annual Meeting of the European Society for Paediatric Infectious Diseases (ESPID). The Hague, The Netherland, 7-11 June 2011, Abstract 1411.

32. Grijalva CG, Poehling KA, Nuorti JP, Zhu Y, Martin SW, Edwards KM, et al. National impact of universal childhood immunization with pneumococcal conjugate vaccine on outpatient medical care visits in the United States. Pediatrics 2006;118: 865-73.

33. Zhou F, Shefer A, Kong Y, Nuorti JP. Trends in acute otitis media-related health care utilization by privately insured young children in the United States, 1997-2004. Pediatrics 2008;121:253-60.

34. Hausdorff WP, Felkin DR, Klugman KP. Epidemiological differences among pneumococcal serotypes. Lancet Infect Dis 2005;5:83-93.

35. Hausdorff WP. The roles of pneumococcal serotypes 1 and 5 in paediatric invasive disease. Vaccine 2007;25:2406-12.

36. Ho PL, Chiu SS, Chow FK, Mak GC, Lau YL. Paediatric hospitalization for pneumococcal diseases preventable by the 7 -valent pneumococcal conjugate vaccine in Hong Kong. Vaccine 2007;25:6837-41.

37. Jefferson T, Ferroni E, Curtale F, Giorgi Rossi P, Borgia P. Streptococcus pneumoniae in western Europe: serotype distribution and incidence in children less than 2 years old. Lancet Infect Dis 2006;6:405-10.

38. Bender JM, Ampofo K, Korgenski K, Daly J, Pavia AT, Mason EO, et al. Pneumococcal necrotizing pneumonia in Utah: does serotype matter ? Clin Infect Dis 2008;46:1346-52.

39. Tyrrell G, Lovgren M, Kellner J, Grimsrud K, Hoang L, Patrick D, et al. Serotype invasive Pneumococcal Investig. Team 2008. A large outbreak of invasive Streptococcus pneumoniae serotype 5 in western Canada. Presented at the ISPPD-6, Reykjavik, Iceland, 8-12 Jun 2008, Abstract Book, p. 50 (S05-O3).

40. Leimkugel J, Adams Forgor A, Gagneux S, Pflüger V, Flierl C, et al. An outbreak of serotype 1 Streptococcus pneumoniae meningitis in Northern Ghana with features that are characteristisc of Neisseria meningitidis meningitis epidemics. J Infect Dis 2005;192:192-9.

41. Yaro S, Lourd M, Traoré Y, Njanpop-Lafourcade BM, Sawadogo A, Sangare L, et al. Epidemiological and molecular characte-ristics of a highly lethal pneumococcal men-ingitis epidemic in Burkina Faso. Clin Infect Dis 2006;43:693-700.

42. Byington CL, Korgenski K, Daly J, Ampofo K, Pavia A, Mason EO. Impact of the pneumococcal conjugate vaccine on pneumo-coccal parapneumonic empyema. Pediatr Inf-ect Dis J 2006;25:250-4.

43. Grijalva CG, Nuorti JP, Arbogast PG, Martin SW, Edwards KM, Griffin MR. Decline in pneumonia admissions after routine childhood immunisation with pneumococcal conjugate vaccine in the USA: A time-series analysis. Lancet 2007;369:1179-86. 
44. Greenberg D, Givon-Lavi N, Newman N, Bar-Ziv J, Dagan R. Nasopharyngeal Carriage of Individual S. pneumoniae Serotypes during Pediatric Pneumonia as a Means to Estimate Serotype Disease Potential. Pediatr Infect Dis J 2011;30:227-33.

45. Patrzałek M, Albrecht P, Sobczynski M. Significant decline in pneumonia admission rate after the introduction of routine $2+1$ dose schedule heptavalent pneumococcal conjugate vaccine (PCV7) in children under 5 years of age in Kielce, Poland. Eur J Clin Microbiol Infect Dis 2010;29:787-92.

46. Simonsen L, Taylor RJ, Young-Xu Y, Haber M, May L, Klugman KP. Impact of pneumococcal conjugate vaccination of infants on pnenumonia and influenza hospitalization and mortality in all age groups in the United States. MBio 2011;2:e00309-10.

47. Black S, Shinefield H, Fireman B, Lewis E, Ray P, Hansen JR, et al. Efficacy, safety and immunogenicity of heptavalent pneumococcal conjugate vaccine in children. Northern California Kaiser Permanente Vaccine Study Center Group. Pediatr Infect Dis J 2000;19:187-95.

48. Eskola J, Kilpi T, Palmu A, Jokinen J, Haapakoski J, Herva E, et al. Efficacy of a pneumococcal conjugate vaccine against acute otitis media. N Engl J Med 2001;344:403-9.

49. Grijalva CG, Poehling KA, Nuorti JP, Zhu Y, Martin SW, Edwards KM, et al. National impact of universal childhood immunization with pneumococcal conjugate vaccine on outpatient medical care visits in the United States. Pediatrics 2006;118:865-73.

50. Wals PD, Carbon M, Sévin E, Deceuninck G, Ouakki M. Reduced physician claims for otitis media after implementation of pneumococcal conjugate vaccine program in the province of Quebec, Canada. Pediatr Infect Dis J 2009;28:e271-5.

51. Jardine A, Menzies RI, Deeks SL, Patel MS, McIntyre PB. The impact of pneumococcal conjugate vaccine on rates of myringotomy with ventilation tube insertion in Australia. Pediatr Infect Dis J 2009;28:761-5.

52. Poehling KA, Szilagyi PG, Grijalva CG, Martin SW, LaFleur B, Mitchel E, et al. Reduction of frequent otitis media and pressure-equalizing tube insertions in children after introduction of pneumococcal conjugate vaccine. Pediatrics 2007;119:707-15.

53. Durando P, Crovari P, Ansaldi F, Sticchi L, Sticchi C, Turello V, et al. Collaborative Group for Pneumococcal Vaccination in Liguria. Vaccine 2009;27:3459-62.

54. Zhou F, Shefer A, Kong Y, Nuorti JP. Trends in acute otitis media-related health care utilization by privately insured young children in the United States, 1997-2004. Pediatrics 2008;121:253-60.

55. Grijalva CG, Nuorti JP, Griffin MR. Antibiotic prescription rates for acute respiratory tract infections in US ambulatory settings. JAMA 2009;302:758-66.

56. Hoberman A, Paradise JL, Shaikh N, Greenberg DP, Kearney DH, Colborn DK, et al. Pneumococcal resistance and serotype 19A in Pittsburgh-area children with acute otitis media before and after introduction of 7-valent pneumococcal polysaccharide vaccine. Clin Pediatr 2011;50:114-20.

57. Dagan R, Givon-Lavi N, Zamir O, Sikuler-Cohen M, Guy L, Janco J, et al. Reduction of nasopharyngeal carriage of Streptococcus pneumoniae after administration of a 9-valent pneumococcal conjugate vaccine to toddlers attending day care centers. J Infect Dis 2002;185:927-36.

58. O'Brien KL, Bronsdon M, Carlone GM, Facklam RR, Schwartz B, Reid RR, et al. Effect of a seven valent pneumococcal conjugate vaccine on nasopharyngeal (NP) carriage among native American infants. Pediatric Academic Societies Annual Meeting. Pediatr Res 2001;49:Abstract 1463, 256A

59. Dagan R, Givon-Lavi N, Zamir O, Sikuler-Cohen M, Guy L, Janco J, et al. Reduction of nasopharyngeal carriage of Streptococcus pneumoniae after administration of a 9-valent pneumococcal conjugate vaccine to toddlers attending day care centers. J Infect Dis 2002;185:927-36.

60. Vestrheim DF, Høiby EA, Aaberge IS, Caugant D. Impact of a pneumococcal conjugate vaccination program on carriage among children in Norway. Clin Vaccine Immunol 2010;17:325-34.

61. Jacobs MR, Dagan R, Bajaksouzian S, Windau AR, Porat N. Validation of Factor 6d Antiserum for Serotyping Streptococcus pneumoniae Serotype 6C. J Clin Microbiol 2010;48:1456-7.

62. Millar EV, Pimenta FC, Roundtree A, Jackson D, Carvalho Mda G, Perilla MJ, et al. Pre and post conjugate vaccine epidemiology of pneumococcal serotype 6C invasive disease and carriage within Navajo and White Mountain Apache communities. Clin Infect Dis 2010;51:1258-65.

63. Porath N, Park IH, Nahm MH, Dagan R. Differential Circulation of Streptococcus pneumoniae Serotype 6C Clones in Two Israeli Pediatric Populations. J Clin Microbiol 2010;48:4649-51.

64. Calix JJ, Dagan R, Pelton SI, Porat N, Nahm MH. Differential occurence of Streptococcus pneumoniae serotype 11E between asymptomatic carriage and invasive pneumococcal disease isolates reflects a unique model of oathogen microevolution. Clin Infect Dis 2012;54:794-9.

65. Hanage WP, Bishop CJ, Huang SS, Stevenson AE, Pelton SI, Lipsitch M, et al. Carried pneumococci in Massachusetts children: The contribution of clonal expansion and serotype switching. Pediatr Inf Dis J 2011;30:302-8.

66. Prymula R, Peeters P, Chrobok V, Kriz P, Novakova E, Kaliskova E, et al. Pneumococcal capsular polysaccharides conjugated to protein D for prevention of acute otitis media caused by both Streptococcus pneumoniae and non-typeable haemophilus influenzae: A randomised double blind efficacy study. Lancet 2006;367:740-8.

67. Hausdorff WP, Bryant J, Paradiso PR, Siber GR. Which pneumococcal serogroups cause the most invasive disease: Implications for conjugate vaccine formulation and use, part I. Clin Infect Dis 2000;30:100-21. 